\title{
Experimental Study of the Flow in the Suction Pipe of A Centrifugal Impeller: Steady Conditions Compared with Fast Start-Up
}

\author{
STÉPHANE BOLPAIRE*, JEAN-PAUL BARRAND, and GUY CAIGNAERT \\ Laboratoire de Mécanique de Lille URA CNRS 1441, Ecole Nationale Supérieure d'Arts et Métiers de Lille, \\ 8, bd Louis XIV - Lille, F-59046, France
}

It is common practice to assume that the pump characteristic expressed in nondimensional variables is not altered by a rotation speed variation. However previous experiments have shown that it is not the case when the rotation speed variation is very fast. In that case, the flow rate increases at a lower gradient than the rotation speed and partial load conditions prevail at the beginning of the start up period. The question is: is there recirculation and if so, what is its axial extent within the suction pipe compared to steady flow conditions at equal nondimensional flow rate? An experimental program has been set up to answer that question in the Laboratoire de Mécanique de Lille.

This paper presents the experimental study of the influence of the operating point on the axial development of the inlet recirculation. The tests were carried out by means of a camcorder, Particles Image Velocimetry and Pressure measurements in the suction pipe, near the inlet of a centrifugal pump, at partial flow rates, in steady conditions and during a fast start up of the pump. All the experiments were performed on the DERAP test bench of the Laboratoire de Mécanique de L'Ecole Nationale Supérieure d'Arts et Métiers de Lille. Standard methods enabled the determining of the recirculation critical flow rate at the pump inlet. Visualization methods showed that the axial growth of the recirculation and the prerotation with the flow rate is considerably reduced during a fast start up compared to steady conditions.

Keywords: Turbomachinery; Radial flow pump; Recirculation; Prerotation; Fast Start-Up; PIV

Nowadays, it is well known that the flow in the suction pipe of an impeller reverses at its outer periphery when a centrifugal pump operates at partial flow rates in steady

Received 16 June 2000; in final form 25 August 2000.

*Corresponding author. Tel.: 33-3-206-22222, Fax: 33-3-20-62-22-40. E-mail: bolpaire@lille.ensam.fr conditions. This phenomenon is accompanied with the appearance of a tangential component of the velocity having its maximum value near the wall pipe and regularly decreasing towards the axis. This swirling flow has the same direction as the impeller rotation and is usually called "prerotation with recirculation". Recirculation appears both on the suction (see Figure 1) and the discharge side of the pump. However, only the inlet part of the phenomenon is considered in this paper.

For high enough flow rates, the flow in the suction pipe can be considered as one dimensional except near the wall pipe, in the boundary layer. When the flow rate is reduced under a value called "critical flow rate" a reverse flow appears and develops along the duct. Its maximum axial extent is obviously reached at shut-off.

The literature contains a lot of studies concerning the onset of the reverse flow. However few authors took an interest in the influence of the operating point on the axial extension of the phenomenon especially during a Fast Start-Up period. This lack of information is comprehensible since the applications are restricted to specific domains (aerospace for example).

Barrand (1996) had already carried out a qualitative analysis of the extension of the recirculation with a first comparison between Fast Start-Up and steady operating conditions. Thus, the results of this first study made possible the setting up of specific techniques, which were characteristic of each type of measurements. Various tests were performed so as to obtain correlation between operating points and the propagation of the phenomenon in the suction pipe.

\section{EXPERIMENTAL DEVICE}

\section{General Overview}

Experiments were carried out in water with a radial flow pump having a dimensionless specific speed $\omega_{\mathrm{s}}$ equal to 


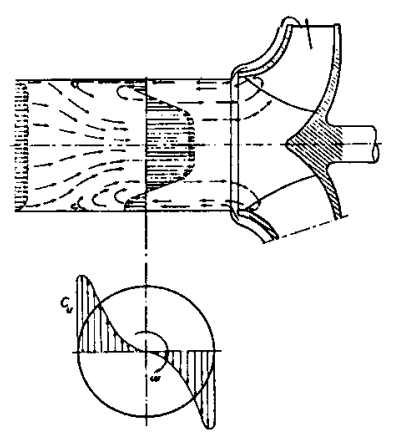

FIGURE 1 Inlet flow arrangement (from Mete Sen, 1980).

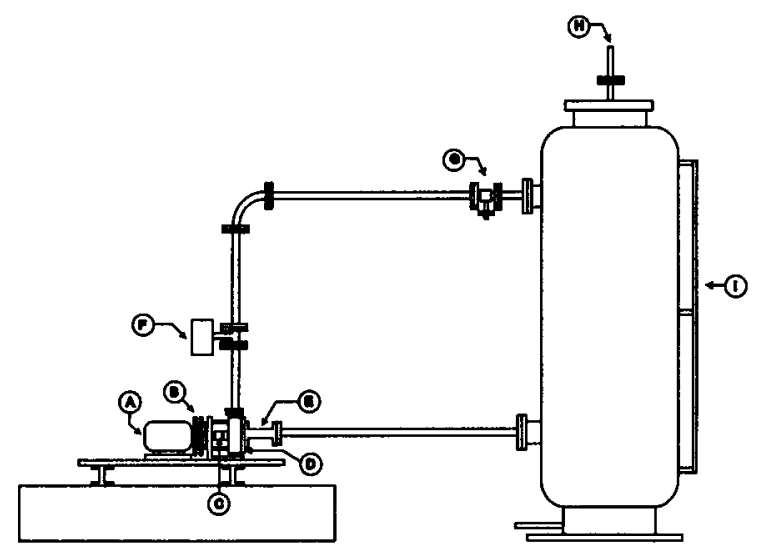

FIGURE 2 General overview of the test rig.

TABLE I Components of the test facilities

\begin{tabular}{ll}
\hline Reference & \multicolumn{1}{c}{ Designation } \\
\hline A & Electric Motor \\
B & Electromagnetic Clutch \\
C & Proximity transducers \\
D & Centrifugal pump \\
E & Measurements section \\
F & Electromagnetic Flowmeter \\
G & Regulation valve \\
H & Pressurization hole \\
I & Level controller \\
\hline
\end{tabular}

0.037 Figure 2 and Table I. The pump is driven by an electric motor allowing very Fast Start-Up when the electromagnetic clutch is rapidly engaged. For example, the rotational speed can rise from 0 to $2900 \mathrm{rpm}$ in $0.3 \mathrm{sec}$. Details of the test rig can be found by Barrand (1996).

The measurement section in the suction pipe consists of a stainless pipe equipped with pressure transducers or of a Plexiglas duct to carry out visualizations or PIV measurements. The proximity transducers mounted on the shaft near the impeller are only used to measure the speed of rotation in the case of a Fast Starting-Up.

\section{Instrumentation}

The study of the axial extent of the recirculation has been performed for two kinds of operating conditions:

- For various steady operating conditions

- During a Fast Start-Up period

Various measurement techniques have been used. PIV and video recordings have been used both in Fast Start-Up period and steady state conditions. Pressure measurements were only used in order to determine the validity of the previous tests.

\section{PIV Principles}

PIV remains a rather difficult technique to implement and requires numbers of adjustments. As shown on Figure 3, velocity maps has been obtained, with use of a PIV technique, in the pump suction pipe. A Plexiglas pipe has been especially manufactured to carry out the measurements. Each image covers a rectangular measurements field: $1280 \times 1024$ pixels corresponding to the pump inlet diameter $(40 \mathrm{~mm})$ and an axial length equal to $92 \mathrm{~mm}$. Measurements have been made with the PIV2100 Dantec System and the Flow Manager software. Hundred instantaneous velocity maps (axial and radial components) have been obtained in the measurements field for each operating condition with a time interval between two maps equal to $1 \mathrm{~s}$.

\section{Visualizations}

\section{Steady Operating Conditions: Micro-Bubbles Injection}

The using of water supersaturated with air coupled with a video-recording make it possible to follow the development of the recirculation. This technique is based upon the expansion of water supersaturated with air. This air-water mixture is injected upstream in the Plexiglas pipe by means of an injector especially designed for the tests as shown on Figure 4.

The distance between the injector and the nosepiece impeller is equal to $330 \mathrm{~mm}$ and the spacing between the

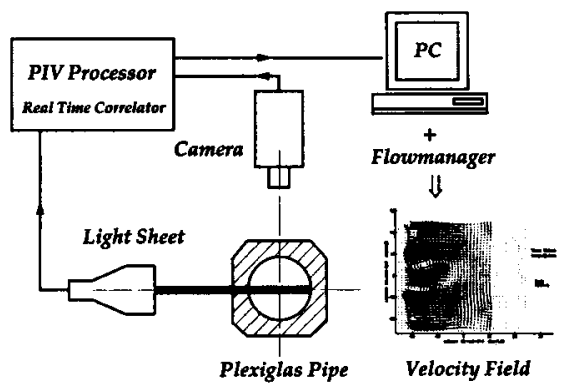

FIGURE 3 PIV acquisition system. 


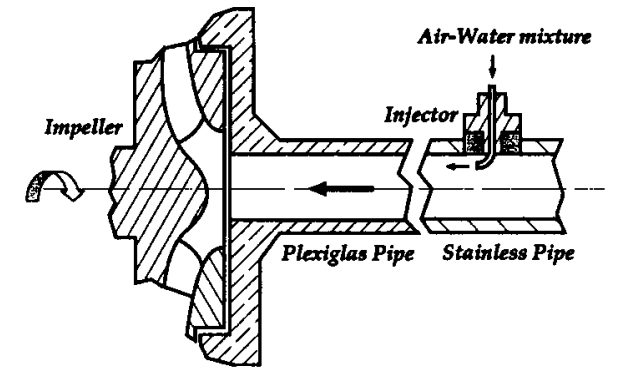

FIGURE 4 Air-water mixture injection principle.

injector and the pipe wall is about $40 \mathrm{~mm}$. Under these conditions, it is possible to follow the flow without perturbing it.

\section{Fast Start-Up: Silicon Sheaths}

The rapid depression occurring during the Starting Up at the upstream of the pump makes the previous method inapplicable. Consequently, we have to use a relatively simple but reliable method: the visualization of the mean direction of silicon sheaths mounted on a frame of brass or partially glued close to the nosepiece impeller at the outer periphery of the suction pipe Figure 5.

This method is coupled with a digital camera whose recordings can reach 24 frames per second with a shutter speed of up to 4 milliseconds. Flow rate and impeller rotation velocity are also measured during the starting up so as to be able to localize each frame with its operating point in terms of rotational speed and flow rate.

In the case of Fast Start-Up, the electromagnetic flowmeter and the tachometric cell are respectively replaced by a method with three-equidistant pressure transducer and a measurement technique with two eccentric mounted on the shaft of the pump.

\section{Pressure Measurements}

Four piezoelectric transducers ${ }^{1}$ have been flush mounted in the suction pipe of the impeller and connected to amplifiers by the means of low noise cables Figure 6 .

This method makes it possible to determine the flow rate for which a transducer becomes sensitive to a local variation of the static pressure generated by the recirculation. Indeed, the appearance of recirculation results in a rapid increase of the level of the local static pressure as it was presented by Hureau (1993). Thus, these recordings allow the characterization of the onset of the phenomenon in the suction pipe of the pump for each measurement section.

\footnotetext{
${ }^{1}$ Kistler 701A.
}

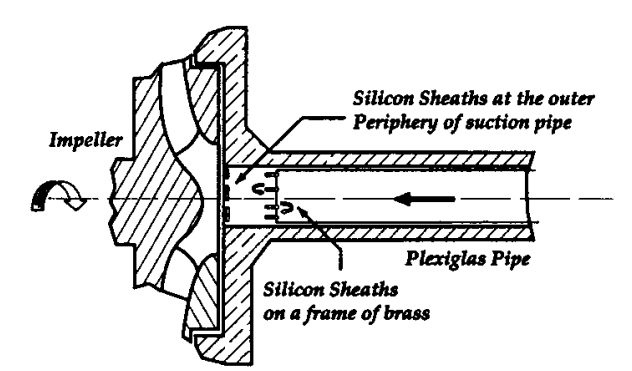

FIGURE 5 Principle of visualization in Fast Start-Up.

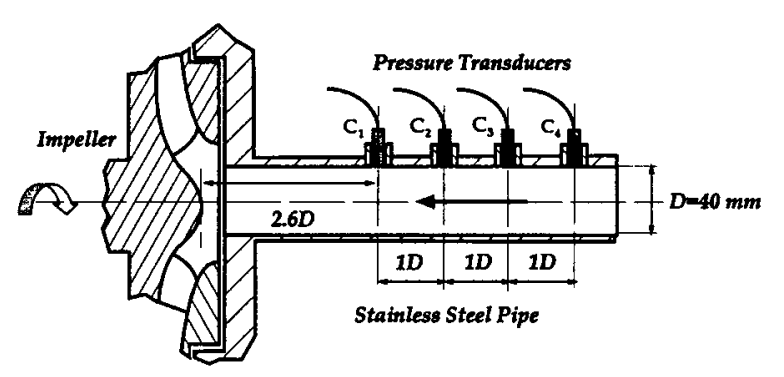

FIGURE 6 Pressure transducers localization.

This method is only applicable during tests in steady conditions and allows the validation of the results obtained by the other techniques.

\section{EXPERIMENTAL RESULTS}

\section{Steady State Results}

The mean static pressures have been recorded in four different axial positions at $1800 \mathrm{rpm}$ and various flow rate. Figure 7 shows the pressure level evolution as a function of the relative flow rate. ${ }^{2}$ Each sudden change of the slope is associated to the appearance of the recirculation in a given position. It is a relatively reliable method to detect the phenomenon. The measurement range is rather restricted because of establishment problems and the minimal spacing between transducers.

The points plotted on Figure 8 reproduce the evolution of the axial development of the reverse flow as a function of the relative flow rate.

The values of the axial extent $(x)$ are divided by the internal diameter of the suction pipe $(D)$ so as to have dimensionless parameters. As the number of pressure taps is limited to four positions, we used a least squares estimation in order to get the evolution of the phenomenon on a greater range. This evolution seems to be exponential

\footnotetext{
${ }^{2}$ Relative flow rate is defined as the ratio of the measured flow rate to the nominal flow rate for the given speed of rotation.
} 


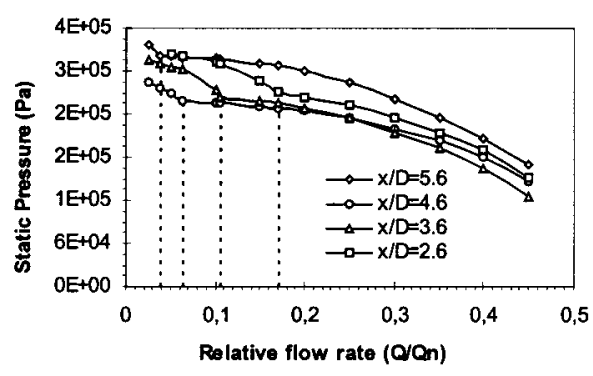

FIGURE 7 Evolution of the static pressure.

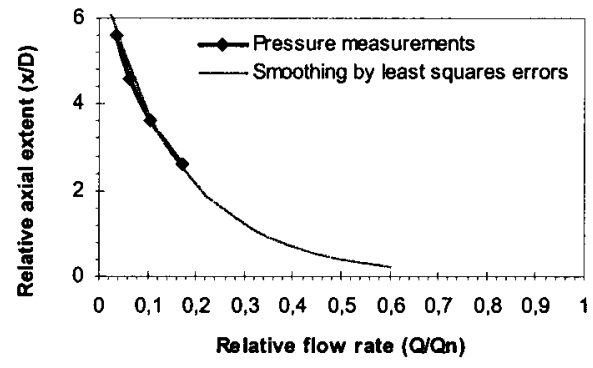

FIGURE 8 Axial extent of the recirculation.

which has been confirmed by Bolpaire (1999) with other experiments. The smoothing curve is voluntarily truncated around $60 \%$ of the nominal flow rate due to the lack of information in this critical area. However, these first results already constitute a good database.

The micro-bubbles injection has been used as a second way in order to study the evolution of the reverse flow with the modification of the operating conditions. Tests have been performed for three speeds of rotation and various flow rates (Figure 10). Results have been compared at $1800 \mathrm{rpm}$ with the pressure measurements so as to validate the method of the micro-bubbles (Figure 9).

Apart from the fact that it is not possible with pressure measurements to get informations for flow rates higher than $20 \%$ of the nominal capacity, one can notice a rather good superposition of the curves on Figure 9 in the low flow rates. This observation seems to provide the obvious proof that it is now possible to follow correctly the

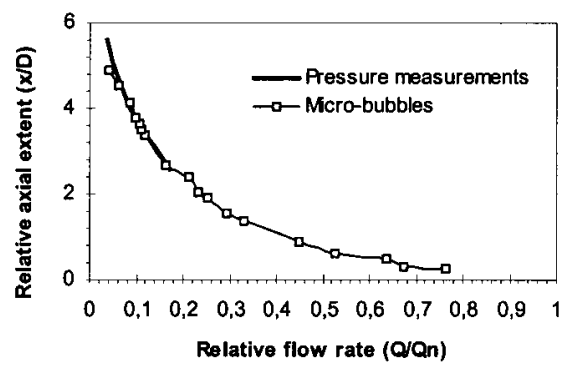

FIGURE 9 Validation of the micro-bubbles technique.

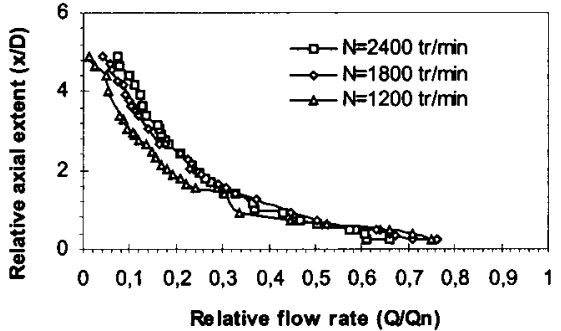

FIGURE 10 Extent of the recirculation as a function of the flow and the speed.

evolution of the flow with a microbubbles technique without perturbing it.

One can notice on these curves an almost identical tendency of evolution with only small differences from one speed to another. This distinction seems to indicate the existence of a slight influence of the speed of rotation on the extent of the phenomenon. A test at constant relative flow rate for different speeds has been set up to verify this hypothesis (Figure 11).

These last results confirm the previous hypothesis: the speed of rotation and the associated Reynolds number have a noticeable effect on the development of inlet recirculation in the suction pipe, especially for very low relative flow rates.

The PIV technique has then been used in order to get more quantitative results in a larger field of the suction pipe. The Figure 12 presents the measurement zone that has been performed. The water has been seeded with

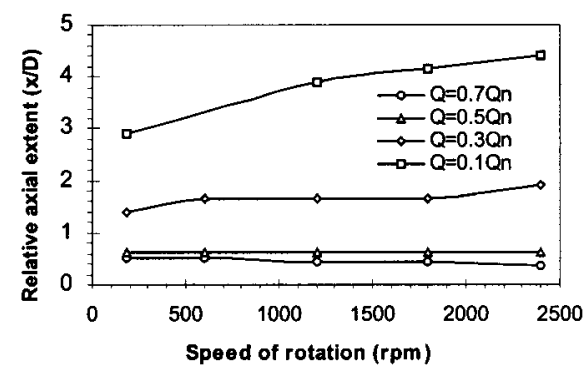

FIGURE 11 Influence of the speed on the recirculation.

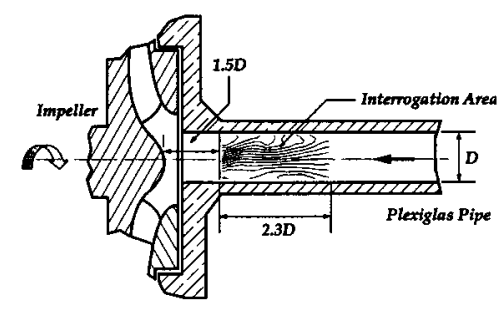

FIGURE 12 PIV measurements. 
Spherical glass balloons $(30 \mu \mathrm{m}$ diameter). The use of a cross-correlation method on $32 \times 32$ pixels windows with a 50 percent overlap allows to get 2600 instantaneous velocity vectors in the field of view.

Figures 13 to 17 present the results for 5 different flow rates for a pump impeller speed of rotation equal to $1800 \mathrm{rpm}$.

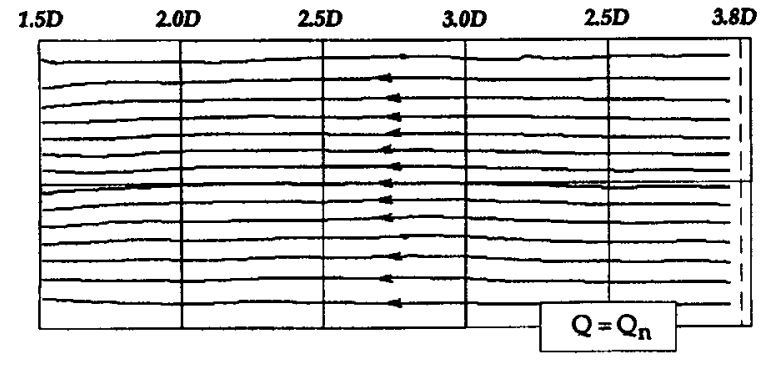

FIGURE 13 Streamlines at the nominal capacity.

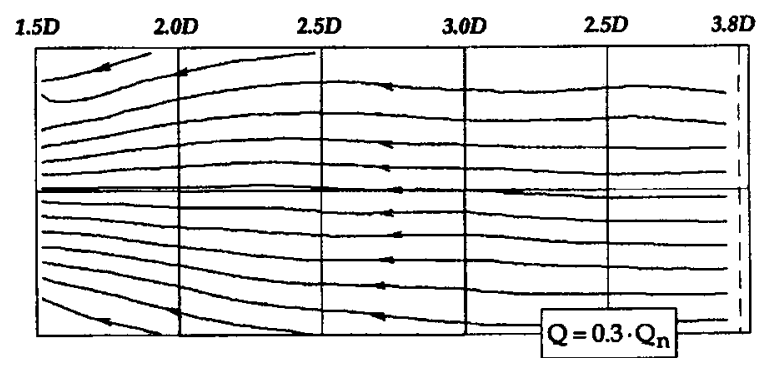

FIGURE 14 Streamlines at $30 \%$ of the relative flow.

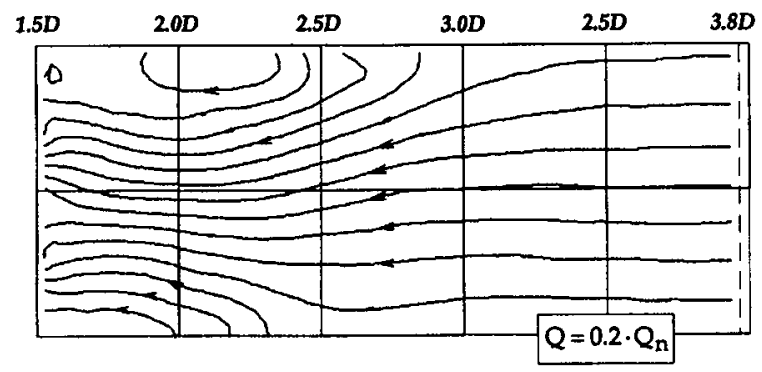

FIGURE 15 Streamlines at $20 \%$ of the relative flow.

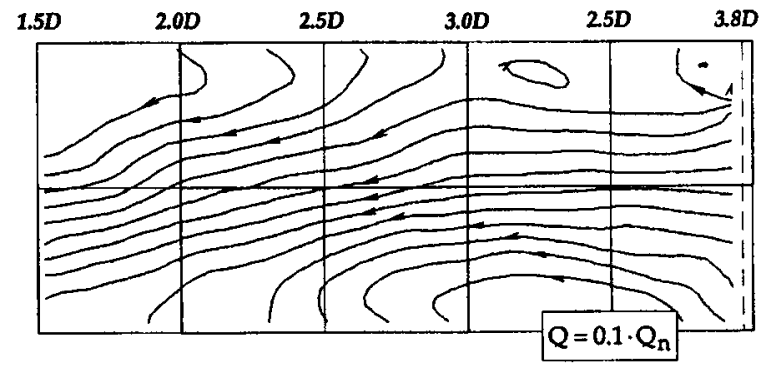

FIGURE 16 Streamlines at $10 \%$ of the relative flow.

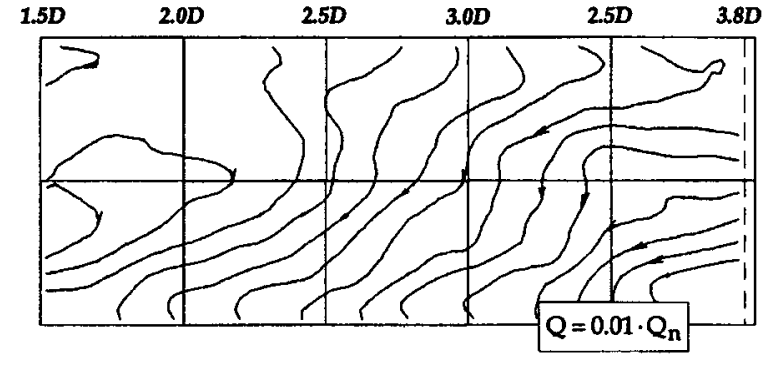

FIGURE 17 Streamlines near shut-off.

Each figure present the streamlines associated to the averaged velocity vectors over the 100 velocity maps.

In agreement with Figure 12, the nosepiece impeller is used as a reference for the axial position. Then, the result becomes dimensionless using the internal diameter of the suction pipe.

When the relative flow rate is approximately equal to the nominal capacity, all the streamlines are parallel to the axial direction. There is no recirculation. If the flow rate is reduced below a certain limit, a reverse flow zone appears with a more or less important axial extent.

Taking into account the limitation of the measurements zone due to the light sheet position and the geometry of the pipe, this limit appears to be non dimensional flow rate equal to $30 \%$ which corresponds to an extent of the phenomenon of about twice the internal diameter of the suction pipe. If the flow is even more reduced, one can notice a development of the disturbed zone.

One clearly sees on these results the appearance of a reversed swirling flow becoming more and more significant as the flow rate is reduced.

On Figures 16 and 17 one notices that the recirculation zone becomes even more extensive. The flow, which was relatively permanent, seems to be now completely random.

The unsteady character of the flow in the suction pipe when the recirculation zone is developing is well established by the examination of a complete sequence of instantaneous velocity maps.

Figure 18 presents the instantaneous streamlines associated to 6 consecutive velocity maps that have been obtained for a relative flow rate equal to 1 percent and impeller speed of rotation equal to $1800 \mathrm{rpm}$. The time interval between two maps is equal to $1 \mathrm{~s}$.

That Figure 18 clearly establishes that recirculation is not a steady phenomenon. This observation could not have been obtained without a PIV method.

In spite of the limited frequency analysis, the PIV technique makes it possible to highlight the pseudo-random character of the recirculation in steady state operating conditions. In order to make the comparison with a Fast Start-Up, the PIV technique requires the use of a trigger function so as to have synchronized measurements. This is 

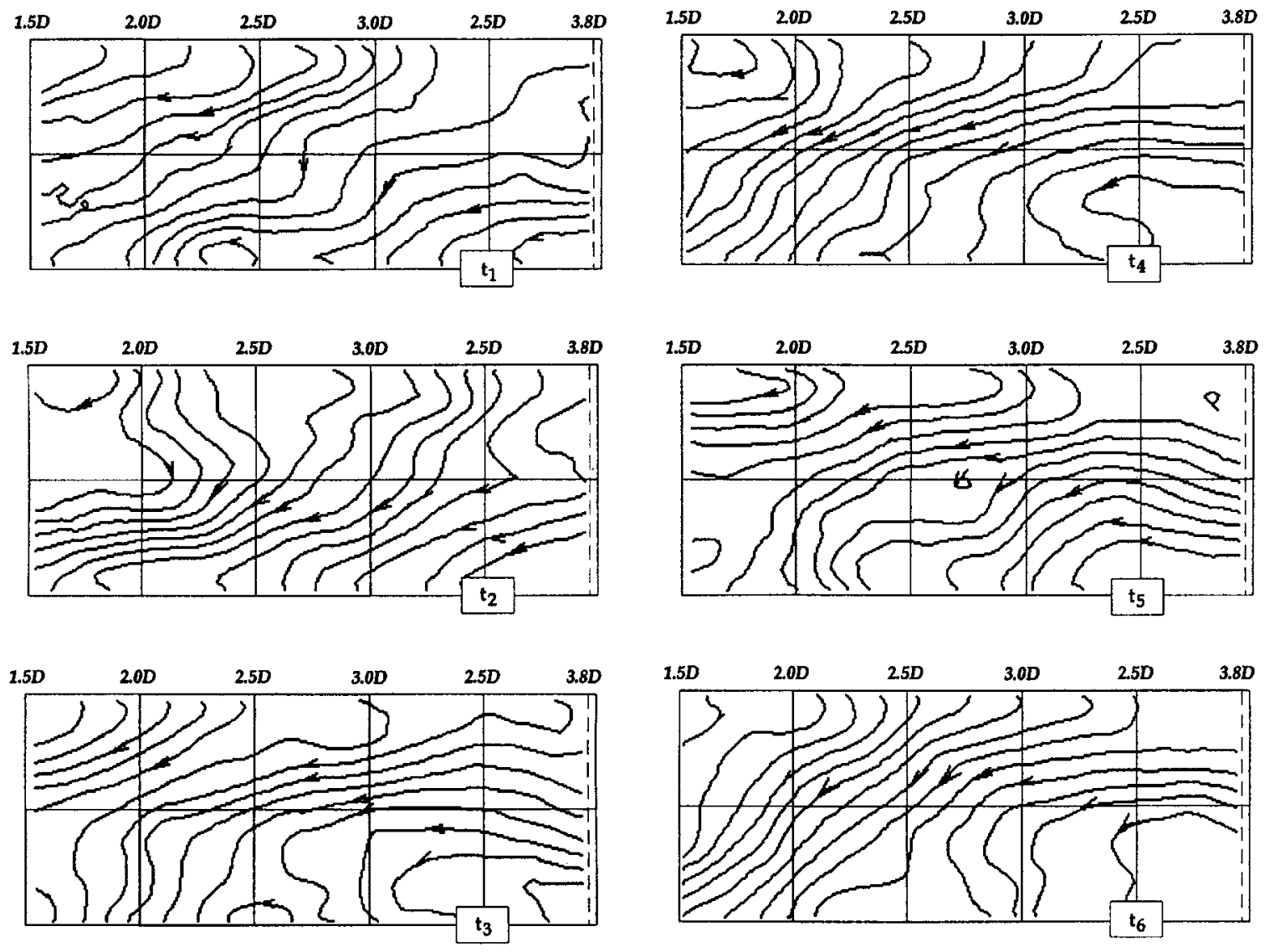

FIGURE 18 Temporal evolution of the recirculation with $1 \%$ of the nominal capacity.

still difficult to manage according to limitation in the frequency of the pulsed lasers. For this kind of measurements, the use of a camcorder remains the most effective solution.

\section{Comparison with a Fast Start-Up Period}

The results presented here refer to tests with the following characteristics:

The final operating point corresponds to the nominal working conditions of the pump. A digital camcorder has been used so as to record frames during the Start-Up. According to the properties of the camera, the beginning of the impeller rotation can be defined with an uncertainty of about $0.2 \mathrm{~s}$.

During the tests, the flow rate and the rotational speed are also measured so as to obtain a link between each frame and the instantaneous operating point Figure 19 and Table II.

At the beginning of the impeller rotation (frame 1) there is no indication of any movement of the flow. On the

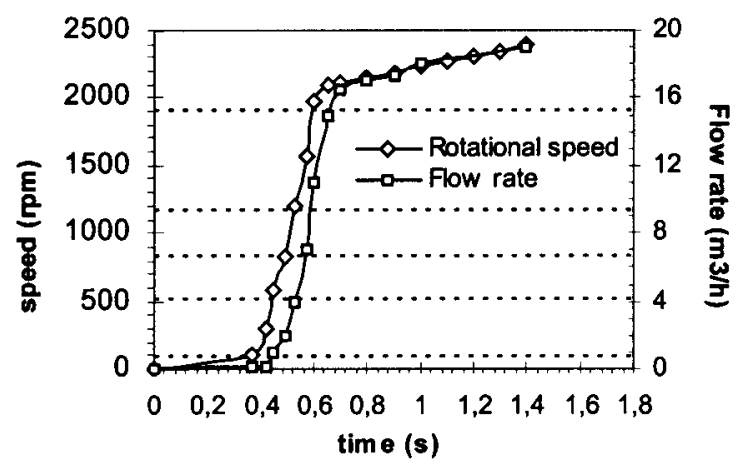

FIGURE 19 Speed and capacity evolution during Start-Up.

second frame, the threads glued at the outer periphery of the pipe (1b) indicate an inverted direction of the flow (frame 2). The third frame clearly indicates prerotation occurrence for two sheaths on the frame of brass $(2 \mathrm{c}, 3 \mathrm{c})$ and also for those located near the inlet pipe (1c). Then, the recirculating zone reaches its maximum extension on the next figure and begins to disappear on frame 4 and finally completely removed on the fifth. The location of the pipe 


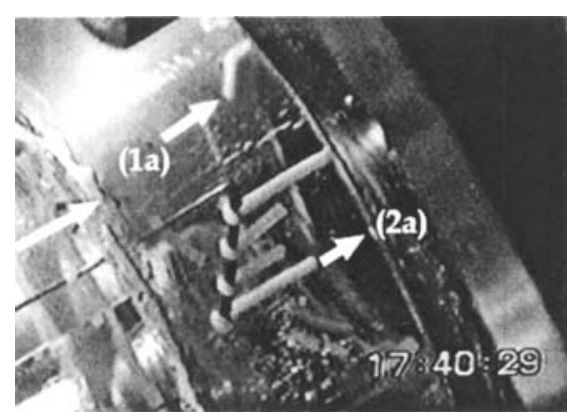

Frame a

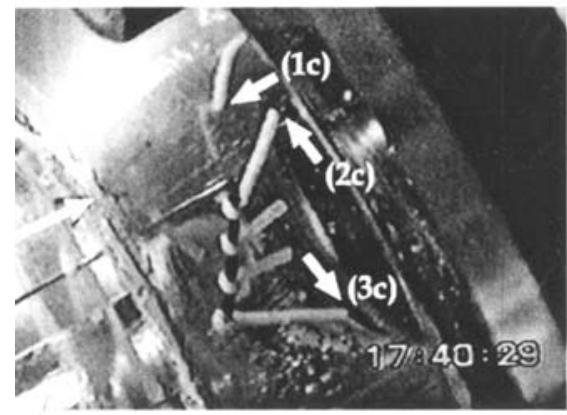

Frame c

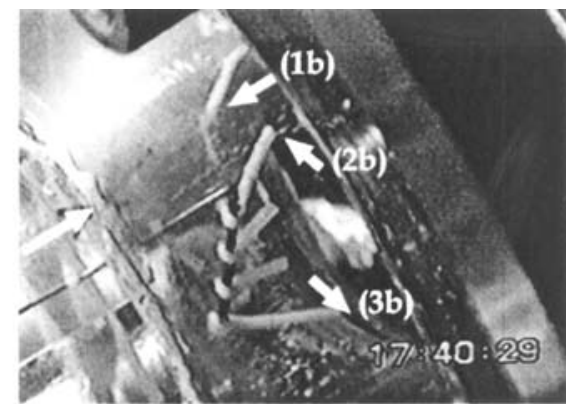

Frame b

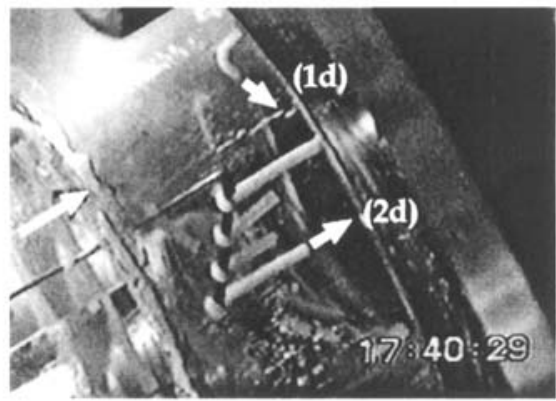

Frame d

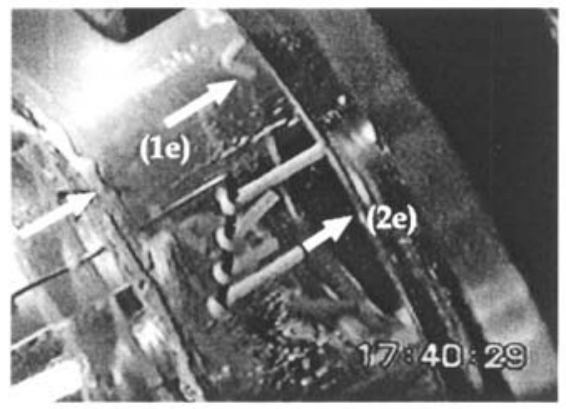

Frame e

FIGURE 20 Show the flow direction.

TABLE II Final characteristics

\begin{tabular}{lc}
\hline Designation & Value \\
\hline Final speed & $2400 \mathrm{rpm}$ \\
Relative Final flow rate & 1 \\
Nominal time of acceleration & $0.3 \mathrm{~s}$. \\
\hline
\end{tabular}

TABLE III Location of frames

\begin{tabular}{llcc}
\hline Ref. of frames & $Q / Q_{n}$ & $N(\mathrm{rpm})$ & $x / D$ \\
\hline $\mathrm{a}$ & 0.13 & 100 & $0<-<0.25$ \\
$\mathrm{~b}$ & 0.16 & 524 & $0.25<-<0.75$ \\
$\mathrm{c}$ & 0.26 & 848 & $>0.75$ \\
$\mathrm{~d}$ & 0.4 & 1181 & $0.25<-<0.75$ \\
$\mathrm{e}$ & 0.72 & 1919 & $0<-<0.25$ \\
\hline
\end{tabular}

makes it possible to determine the exact axial position of the extension of the reverse flow zone for each frame (see Table III).

The results clearly indicate that the recirculating and the prerotating zone is very much influenced by the operating conditions especially during a Starting Up. During this period, the extension of the phenomenon is smaller than in steady conditions. These tests confirm some previous hypotheses put forth by Barrand et al. (1996).

\section{CONCLUSION}

The existence of a prerotating velocity field during a Fast Start-Up has been clearly demonstrated using various 
measurement techniques. These new results confirm some previous hypotheses put forth by Barrand et al. (1996). Our results show that the reverse flow zone in the suction pipe of a centrifugal pump has a very much lower extension during the starting up compared to the equivalent steady states. Apart from the fact it was shown with PIV that the phenomenon is not steady, the main conclusion is that the instantaneous flow cannot be easily extrapolated from data obtained in the steady state.

Finally, this new information must be taken into account in the development of the characteristics and the hydraulic losses of centrifugal machines during unsteady operations.

\section{Acknowledgment}

The authors take this opportunity to thanks DANTEC company for their participation to PIV measurements, Centre National d'Etudes Spatiales and Electricité de France companies for their financial support and participation to this research project.

\section{NOMENCLATURE}

$D \quad$ Internal pipe diameter $(\mathrm{mm})$

$N \quad$ Rotational speed (rev/mm)

$Q$ flow rate $\left(\mathrm{m}^{3} / \mathrm{h}\right)$

$Q_{n} \quad$ Nominal flow rate $\left(\mathrm{m}^{3} / \mathrm{h}\right)$

$\omega_{\mathrm{S}}$ Dimensionless specific speed

\section{REFERENCES}

Barrand, J.-P. and Picavet, A. (1996) Qualitative flow visualizations during fast start up of centrifugal pumps, Proceedings of the XVIII IAHR Symposium on Hydraulic Machinery and Cavitation, Group 5, II, $671-680$

Bolpaire, S. and Barrand, J.-P. (1999) Experimental study of the flow in the suction pipe of a centrifugal impeller at partial flow rates in unsteady conditions, Journal of pressure and vessel technology, Transactions of ASME, 121, 290-295.

Hureau, F., Kermarec, J., and Foucher, D. (1993) Etude de l'écoulement instationnaire dans une pompe centrifuge fonctionnant à débit partiel, Pump noise and vibrations, 1st International Conference, pp. 51-58.

Mete Sen, H. (1980) Study of inlet flow of centrifugal pumps at partial flow rates, Ph.D. Thesis, Brussels University. 

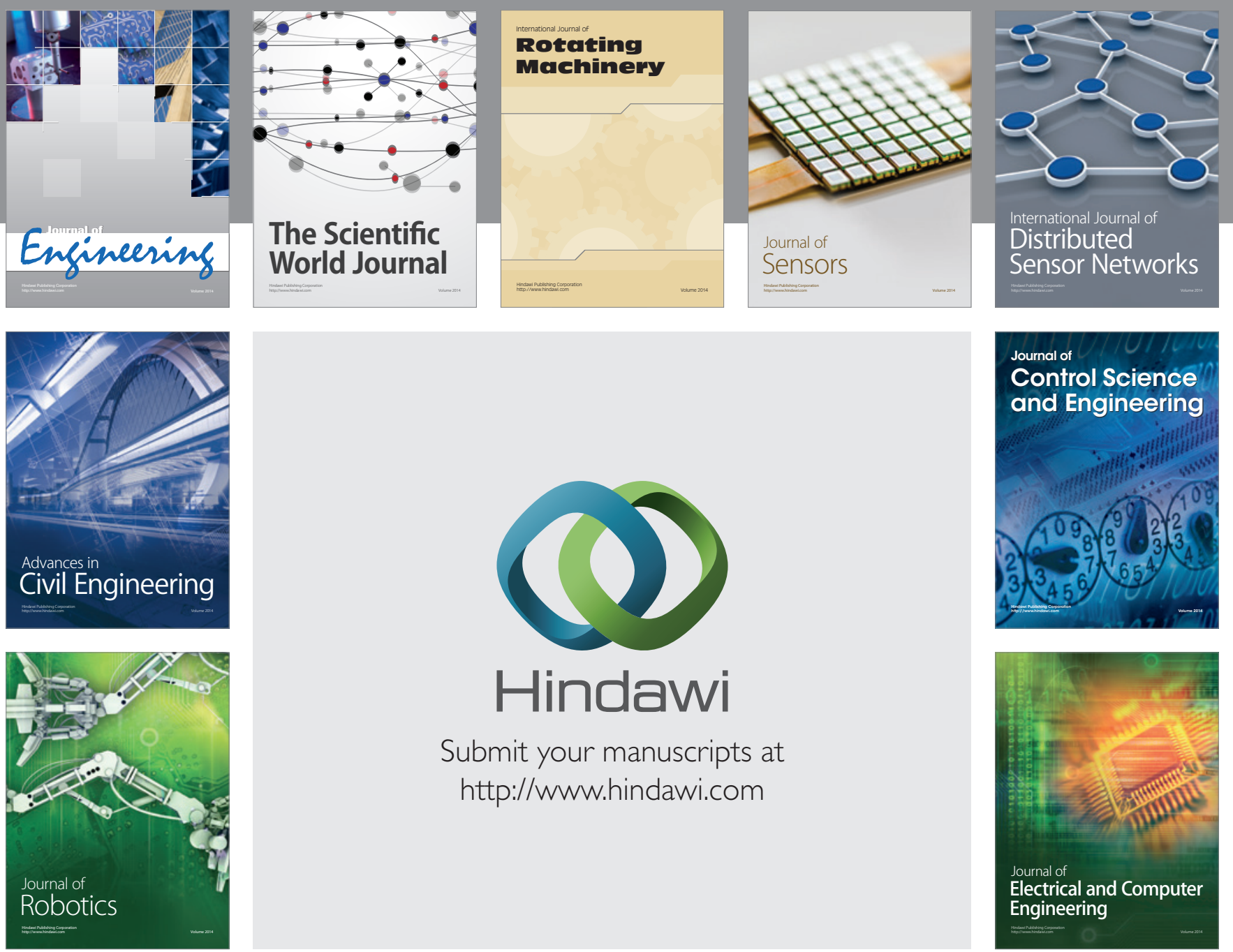

Submit your manuscripts at

http://www.hindawi.com
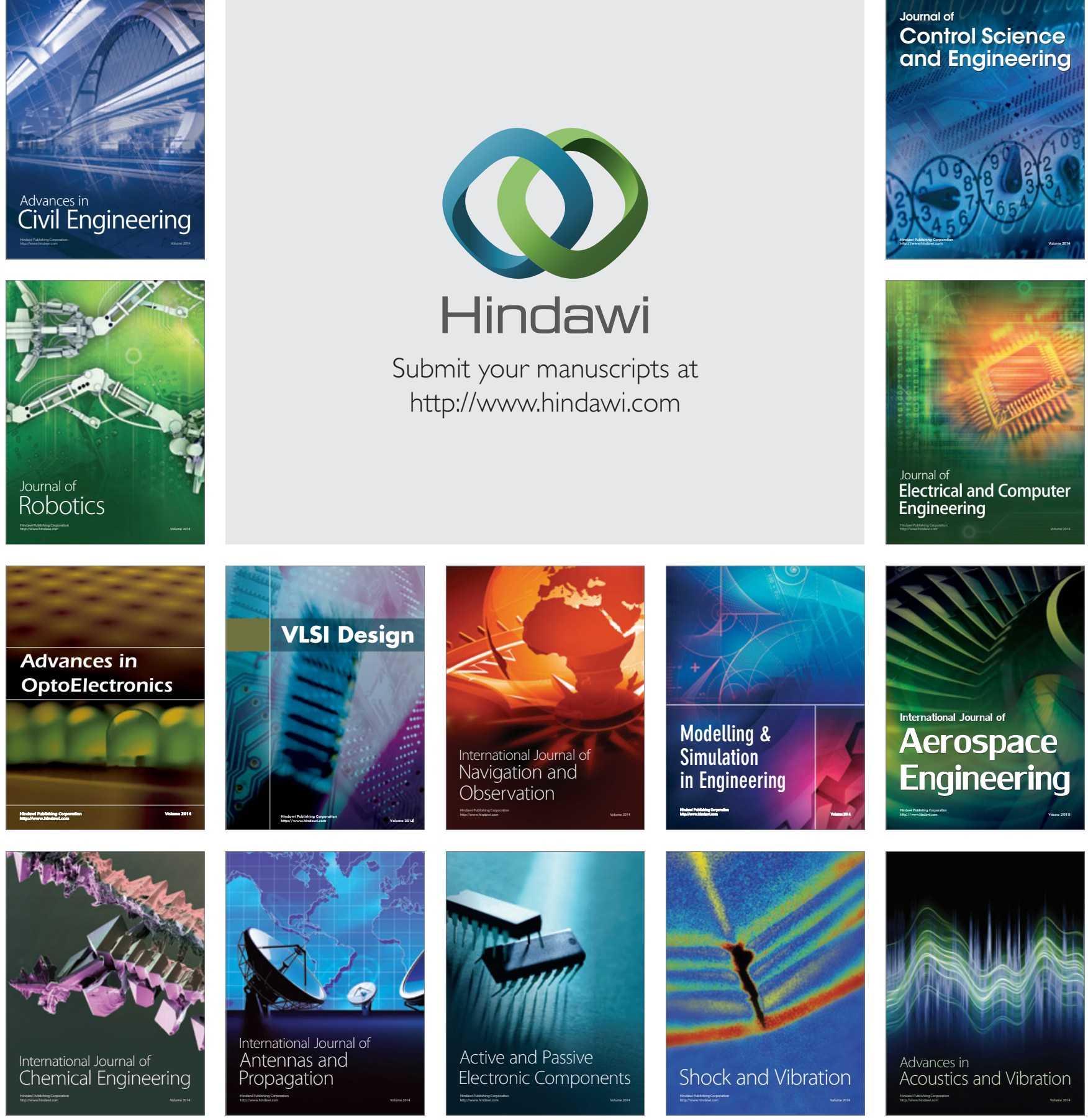